\title{
Semipalmated Sandpiper Calidris pusilla in Brazil: occurrence away from the coast and a new record for the central-west region
}

\author{
Karla Dayane de Lima Pereira ${ }^{1,3}$ \& Jayrson Araújo de Oliveira ${ }^{2}$ \\ Programa Integrado de Estudos da Fauna da Região Centro Oeste do Brasil (FaunaCO), Instituto de Ciências Biológicas, Universidade Federal de \\ Goiás, Goiânia, GO, Brazil. \\ 2 Departamento de Morfologia, Instituto de Ciências Biológicas, Universidade Federal de Goiás, Goiânia, GO, Brazil. \\ 3 Corresponding author: karla.biologia@yahoo.com.br
}

Received on 27 March 2019. Accepted on 16 September 2019.

\begin{abstract}
The Semipalmated Sandpiper, Calidris pusilla, is a Western Hemisphere migrant shorebird for which Brazil forms an internationally important contranuptial area. In Brazil, the species main contranuptial areas is along the Atlantic Ocean coast, in the north and northeast regions. In addition to these primary contranuptial areas, there are also records of vagrants widely distributed across Brazil. Here, we review the occurrence of vagrants of this species in Brazil, and document a new record of C. pusilla for the central-west region and a first occurrence for the state of Goiás.
\end{abstract}

KEY-WORDS: geographical distribution, Nearctic migrant, shorebird, state of Goiás, vagrant.

The Semipalmated Sandpiper Calidris pusilla (Linnaeus, 1766) is a migratory shorebird species that breeds in the Arctic and sub-Arctic regions of Alaska and Canada (Andres et al. 2012, IUCN 2019). Every year, as the northern autumn approaches, Arctic populations fly from 3000 to $4000 \mathrm{~km}$ to South America (Hicklin \& Gratto-Trevor 2010).

In Brazil, C. pusilla occurs during all months of the year, but with very few records during the boreal winter (eBird 2019, GBIF 2019, SNA 2019, WikiAves 2019). This species uses the Atlantic route, occurring from the coastal zone of the state of Amapá to the state of Rio Grande do Sul, where the main contranuptial areas with population concentrations of this bird are located along of the Reentrâncias Paraenses and Maranhenses and in the states of Amapá and Pernambuco (Rodrigues 2007, Carvalho \& Rodrigues 2011, Rodrigues et al. 2015, SNA 2019, GBIF 2019). In the southeast and south regions, only a small population of this species is observed (Harrington et al. 1986, Resende et al. 1989, Barbieri et al. 2013, eBird 2019, GBIF 2019, WikiAves 2019).

In addition to the primary contranuptial areas mentioned above, there are also records of vagrants widely distributed inland in Brazil (Table 1). Based on the available information, five previous records of $C$. pusilla were found for the central-west region, of which three were obtained in the municipality of Poconé, state of Mato Grosso (Cintra 2011, Levatich \& Padilha 2019) and two in the municipality of Corumbá, state of Mato Grosso do Sul (Serrano 2010, Tubelis \& Tomas 2003). However, there is no evidence that these records have been correctly identified, as individuals appear not to have been collected and sent to a scientific collection, nor are images available to validate records.

On 11, 12 and 13 November 2018, an adult specimen of C. pusilla was observed in an area denominated "Lago da Piscicultura Frutos D'Água" in the municipality of Goiânia, state of Goiás, Brazil (16²3'25"S; 4918'48'"W, $719 \mathrm{~m}$ a.s.l.). During three consecutive days, C. pusilla was sighted in the late afternoons, foraging most of the time along with a maximum of four White-rumped Sandpipers Calidris fuscicollis (Vieillot, 1819) and with two Solitary Sandpipers Tringa solitaria Wilson, 1813 (Fig. 1). Very similar to the congeneric C. mauri and $C$. minutilla, which were not present at the site, the C. pusilla individual was safely identified through high-quality photographic records. Compared to C. minutilla, its size was larger, with gray upper plumage and black legs, thick beak with absence of slight (Barnett et al. 2004, Lees et al. 2013). In contrast to $C$. mauri, the head was smaller and less frontal in shape, the beak had a thick tip and the center of the chest was not striated (Barnett et al. 2004, eBird 2019). Digital vouchers were uploaded to WikiAves (WA3179032). 
Table 1. Review of the occurrence of vagrant Calidris pusilla in Brazil. Location: state, municipality, place description and geographical coordinates - latitude (S), longitude (W). Verifiable evidences: P - photograph, S - preserved specimen, $\mathrm{R}$ - recovery of marked individual, $\mathrm{V}$ - visually observed; - no specimen available. $n=$ Number of individuals. Specimens: number of tumble and their respective collections (CLO:EBIRD - eBird, Cornell Lab of Ornithology; UNICAMP:FNJV - Fonoteca Neotropical "Jacques Vielliard"; WA - WikiAves).

\begin{tabular}{|c|c|c|c|c|c|}
\hline Location & Date & $\begin{array}{l}\text { Verifiable } \\
\text { evidences }\end{array}$ & $n$ & Specimens & Reference \\
\hline $\begin{array}{l}\text { Amazonas, Manaus, little lake in } \\
\text { Careiro }\end{array}$ & $\begin{array}{l}29 \text { March } \\
1987\end{array}$ & $\mathrm{~V}$ & 1 & - & Stotz et al. 1992 \\
\hline $\begin{array}{l}\text { Amazonas, Iranduba, Marchantaria } \\
\text { Island }\end{array}$ & $\begin{array}{l}16 \text { February } \\
1989\end{array}$ & $\mathrm{~V}$ & 8 & - & Stotz et al. 1992 \\
\hline $\begin{array}{l}\text { Pará, Terra Santa, Mexiana Island } \\
\left(1^{\circ} 57^{\prime} \mathrm{S} ; 56^{\circ} 21^{\prime} \mathrm{W}\right)\end{array}$ & - & - & 1 & - & $\begin{array}{l}\text { Hellmayr \& } \\
\text { Conover } 1948 \text { apud } \\
\text { Serrano } 2010\end{array}$ \\
\hline $\begin{array}{l}\text { Tocantins, Itacajá, near the } \\
\text { municipality of Recursolândia }\end{array}$ & $\begin{array}{l}31 \text { October } \\
2000\end{array}$ & $\mathrm{~V}, \mathrm{R}$ & 1 & - & $\begin{array}{l}\text { CEMAVE } 2007 \\
\text { apud Serrano } 2010\end{array}$ \\
\hline $\begin{array}{l}\text { Maranháo, Timon, crown of the } \\
\text { Paranaíba River }\end{array}$ & $\begin{array}{l}2 \text { November } \\
2017\end{array}$ & $\mathrm{~V}, \mathrm{P}$ & 2 & WA2765260 & Galvão 2017 \\
\hline $\begin{array}{l}\text { Ceará, São Benedito, Ibiapaba } \\
\left(4^{\circ} 00^{\prime} S ; 41^{\circ} 00^{\prime} \mathrm{W}\right)\end{array}$ & $\begin{array}{l}08 \text { January } \\
1929\end{array}$ & - & 1 & - & $\begin{array}{l}\text { Hellmayr \& } \\
\text { Conover } 1948 \text { apud } \\
\text { Serrano } 2010\end{array}$ \\
\hline $\begin{array}{l}\text { Ceará, Sobral, Lagoa das Marrecas } \\
\left(3^{\circ} 41^{\prime} 15.8^{\prime \prime} ; ; 40^{\circ} 19^{\prime} 51.3^{\prime \prime W}\right)\end{array}$ & $\begin{array}{l}13 \text { October } \\
2018\end{array}$ & $\mathrm{~V}$ & 11 & CLO:EBIRD:OBS666659024 & $\begin{array}{l}\text { Levatich \& Padilha } \\
\qquad 2019\end{array}$ \\
\hline $\begin{array}{l}\text { Pernambuco, Petrolina } \\
\text { (9²4'18.1"S; 4032'10.0"W) }\end{array}$ & $\begin{array}{l}15 \text { February } \\
2003\end{array}$ & V & 1 & CLO:EBIRD:OBS72752146 & $\begin{array}{l}\text { Levatich \& Padilha } \\
\quad 2019\end{array}$ \\
\hline $\begin{array}{l}\text { Pernambuco, Serra Talhada, Açude } \\
\text { do Saco }\end{array}$ & $\begin{array}{l}08 \text { December } \\
1973\end{array}$ & $S$ & 1 & UNICAMP:FNJV:0000000656 & Toledo 2019 \\
\hline $\begin{array}{l}\text { Bahia, Juazeiro, Caatinga Forest } \\
\left.\text { east ( } 9^{\circ} 30^{\prime} 52.4^{\prime \prime} \text {; } 40^{\circ} 07^{\prime} 23.6^{\prime \prime} \mathrm{W}\right)\end{array}$ & $\begin{array}{l}15 \text { February } \\
2003\end{array}$ & V & 2 & CLO:EBIRD:OBS471407770 & $\begin{array}{l}\text { Levatich \& Padilha } \\
\quad 2019\end{array}$ \\
\hline $\begin{array}{l}\text { Bahia, Mata de São João, } \\
\text { Sapiranga Reserve (12³4'04.6"S; } \\
\left.38^{\circ} 02^{\prime} 14.8^{\prime \prime W}\right)\end{array}$ & $\begin{array}{l}25 \text { January } \\
2013\end{array}$ & V & 1 & CLO:EBIRD:OBS499778311 & $\begin{array}{l}\text { Levatich \& Padilha } \\
\quad 2019\end{array}$ \\
\hline $\begin{array}{l}\text { Bahia, Santa Rita de Cássia, Preto } \\
\text { River }\left(11^{\circ} 00^{\prime} S ; 44^{\circ} 32^{\prime} \mathrm{W}\right)\end{array}$ & 04 May 1910 & - & 1 & - & $\begin{array}{l}\text { Hellmayr \& } \\
\text { Conover } 1948 \text { apud } \\
\text { Serrano } 2010\end{array}$ \\
\hline $\begin{array}{l}\text { Santa Catarina, Santa Rosa de } \\
\text { Lima, Pousada Doce Encanto } \\
\left(28^{\circ} 02^{\prime} 00.2^{\prime \prime} S ; 49^{\circ} 09^{\prime} 00.9^{\prime \prime} \mathrm{W}\right)\end{array}$ & $\begin{array}{l}18 \text { November } \\
2017\end{array}$ & $\mathrm{~V}$ & 1 & CLO:EBIRD:OBS550251697 & $\begin{array}{l}\text { Levatich \& Padilha } \\
\quad 2019\end{array}$ \\
\hline $\begin{array}{l}\text { Mato Grosso, Poconé, Pantanal of } \\
\text { Poconé }\end{array}$ & $\begin{array}{l}\text { between } 1982 \\
\text { and } 1986\end{array}$ & - & - & - & Cintra 2011 \\
\hline $\begin{array}{l}\text { Mato Grosso, Poconé, Pousada } \\
\text { Curicaca } \\
\left(16^{\circ} 30^{\prime} 16.0^{\prime \prime} S ; 56^{\circ} 40^{\prime} 30.7^{\prime \prime} \mathrm{W}\right)\end{array}$ & $\begin{array}{l}11 \text { September } \\
2013\end{array}$ & $\mathrm{~V}$ & 1 & CLO:EBIRD:OBS224967965 & $\begin{array}{l}\text { Levatich \& Padilha } \\
\quad 2019\end{array}$ \\
\hline $\begin{array}{l}\text { Mato Grosso, Poconé, Porto Jofre } \\
\left(17^{\circ} 21^{\prime} 52.3^{\prime \prime S} \text {; 566' } 40.4^{\prime} \mathrm{W}\right)\end{array}$ & $\begin{array}{l}04 \text { September } \\
\quad 2017\end{array}$ & $\mathrm{~V}$ & 1 & CLO:EBIRD:OBS668324309 & $\begin{array}{l}\text { Levatich \& Padilha } \\
\quad 2019\end{array}$ \\
\hline $\begin{array}{l}\text { Mato Grosso do Sul, Corumbá, } \\
\text { Nhecolândia in Campinas Farm }\end{array}$ & $\begin{array}{c}\text { October } 1988 \\
\text { and October } \\
1989\end{array}$ & $\mathrm{~V}$ & - & - & Serrano 2010 \\
\hline $\begin{array}{l}\text { Mato Grosso do Sul, Corumbá, } \\
\text { Nhumirim Farm } \\
\left(18^{\circ} 59^{\prime} \mathrm{S} ; 56^{\circ} 39^{\prime \prime} \mathrm{W}\right)\end{array}$ & $\begin{array}{l}\text { between } 1991 \\
\text { and } 1992\end{array}$ & V & - & - & $\begin{array}{l}\text { Tubelis \& Tomas } \\
\qquad 2003\end{array}$ \\
\hline
\end{tabular}



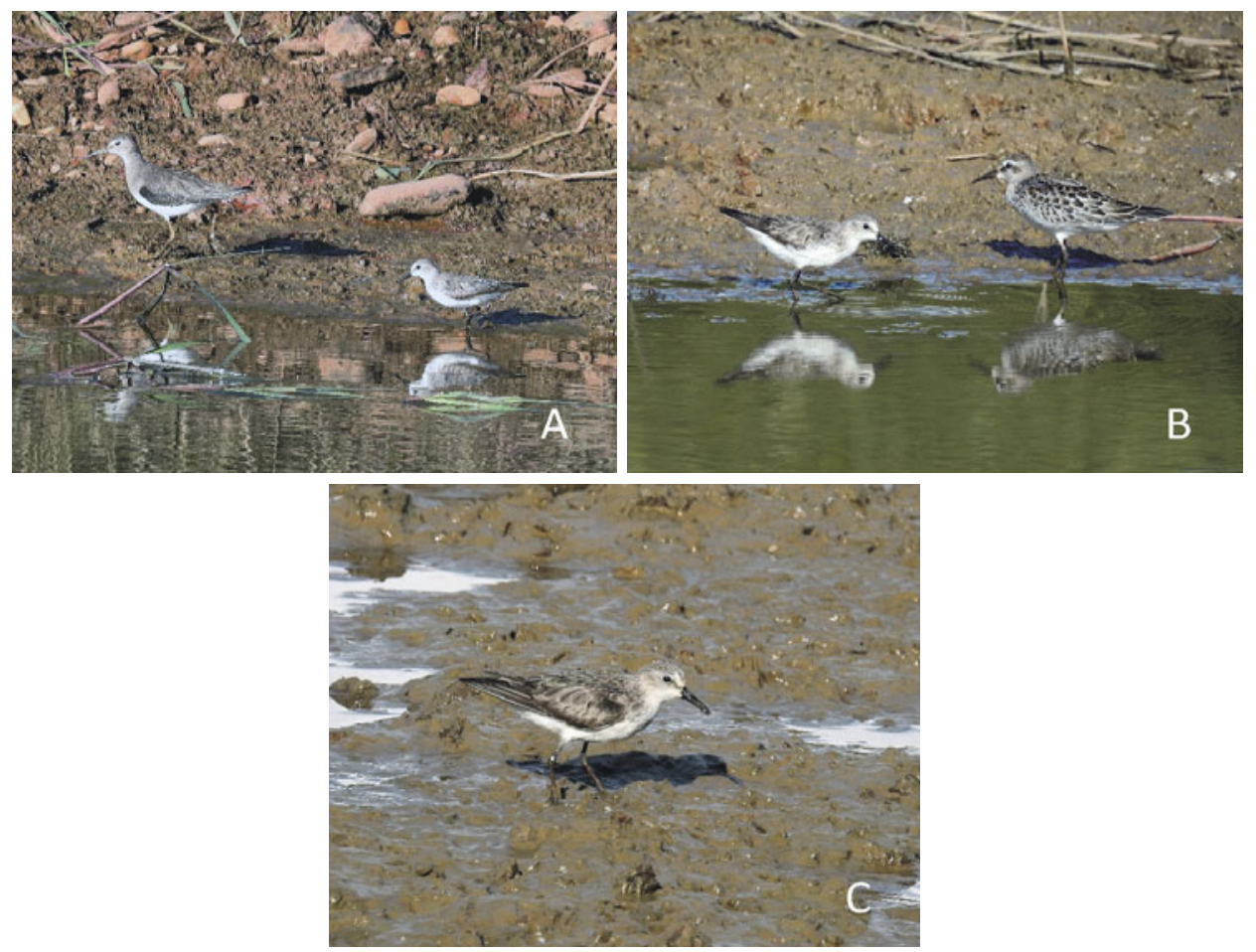

Figure 1. (A) Adult of Calidris pusilla foraging next to adult individual of Tringa solitaria, on 11 November 2018; (B) C. pusilla foraging next to an individual of Calidris fuscicollis, on 12 November 2018; (C) C. pusilla observed on 13 November 2018. All records made in Goiânia, Goiás state, Brazil. Photo author: J.A. Oliveira.

We reviewed the records of vagrants of $C$. pusilla in Brazil and found five previous observations for central Brazil, but there are not enough evidences to confirm the correctness of the identification of these records (Table 1). Thus, here we document a new occurrence of $C$. pusilla for the central-west region and a first record for the state of Goiás.

A bird of international conservation concern, $C$. pusilla is classified as "Near Threatened", due to ongoing population declines (IUCN 2019) linked to several physiological, ecological and human-related challenges, such as: species needs to perform periodic scales for resting and foraging; good habitat conditions in both breeding and contranuptial areas; availability of food resources; competition in foraging environment; predation pressure; human disturbances (Skagen 2006, Burger et al. 2007, IUCN 2019).

\section{ACKNOWLEDGEMENTS}

We would like to thank Alexander C. Lees and Túlio Dornas for helping with the species identification. We also thank Jéssica Vieira Teixeira for her helpful suggestions on the manuscript. We thanks to the researchers who provided their data deposited in the "Sistema Nacional de Anilhamento de Aves Silvestres (SNA)".

\section{REFERENCES}

Andres B.A., Gratto-Trevor C., Hicklin P., Mizrahi D., Morrison R.I.G. \& Smith P.A. 2012. Status of the Semipalmated Sandpiper. Waterbirds 35: 146-148.

Barbieri E., Delchiaro R.T.C. \& Branco J.O. 2013. Flutuaçóes mensais na abundância dos Charadriidae e Scolopacidae da praia da Ilha Comprida, São Paulo, Brasil. Biota Neotropica 13: 268-277.

Barnett J.M., Minns J., Kirwan G.M. \& Remold H. 2004. Informaçôes adicionais sobre as aves dos estados do Paraná, Santa Catarina e Rio Grande do Sul. Ararajuba 12: 53-56.

Burger J., Carlucci S.A., Jeitner C.W. \& Niles L. 2007. Habitat choice, disturbance, and management of foraging shorebirds and gulls at a migratory stopover. Journal of Coastal Research 23: 1159-1166.

Carvalho D.L. \& Rodrigues A.A.F. 2011. Spatial and temporal distribution of migrant shorebirds (Charadriiformes) on Caranguejos Island in the Gulf of Maranháo, Brazil. Revista Brasileira de Ornitologia 19: 486-492.

Cintra R. 2011. Pantanal de Poconé, p. 193-198. In: Valente R.M., Silva J.M.C., Straube F.C. \& Nascimento J.L.X. (eds.). Conservação de aves migratórias Neárticas no Brasil. Belém: Conservation International.

eBird. 2019. eBird: an online database of bird distribution and abundance [web application]. Ithaca. http://www.ebird.org (Access on 21 August 2019).

Galvão J. 2017. [WA2765260, Calidris pusilla (Linnaeus, 1766)]. www.wikiaves.com/2765260 (Access on 21 August 2019).

GBIF. 2019. Calidris pusilla. Global Biodiversity Information Facility. http://www.gbif.org/what-is-gbif (Access on 21 August 2019).

Harrington B.A., Antas P.T.Z. \& Silva F. 1986. Northward shorebird migration on the Atlantic coast of southern Brazil. Vida Silvestre Neotropical 1: 45-54. 
Hicklin P. \& Gratto-Trevor C.L. 2010. Semipalmated Sandpiper (Calidris pusilla), v. 2.0. In: Poole A.F. (ed.). The birds of North America. Ithaca: Cornell Lab of Ornithology. https://doi. org/10.2173/bna.6 (Access on 21 August 2019).

IUCN. 2019. Calidris pusilla. The IUCN red list of threatened species, $v$. 2018.2. http://www.iucnredlist.org (Access on 21 August 2019).

Lees A.C., Moura N.G., Andretti C.B., Davis B.J.W., Lopes E.V., Henriques L.M.P., Aleixo A., Barlow J., Ferreira J. \& Gardner T.A. 2013. One hundred and thirty-five years of avifaunal surveys around Santarém, central Brazilian Amazon. Revista Brasileira de Ornitologia 21: 16-57.

Levatich T. \& Padilla F. 2019. EOD - eBird Observation Dataset. Cornell Lab of Ornithology. Occurrence dataset. https://doi.org/10.15468/ aomfnb accessed via GBIF.org (Access on 21 August 2019).

Resende S.L., Leeuwenberg F. \& Harrington B.A. 1989. Biometrics of Semipalmated Sandpipers Calidris pusilla in southern Brazil. Wader Study Group Bulletin 55: 25-26.

Rodrigues A.A.F. 2007. Priority areas for conservation of migratory and resident waterbirds on the coast of Brazilian Amazonia. Revista Brasileira de Ornitologia 15: 209-218.

Rodrigues A.A.F., Lopes A.T.L., Gonçalves E.C. \& Schneider M.P.C. 2015. Spring migration of Semipalmated Sandpiper Calidris pusilla in the Amazonian coast of Brazil. Ornithologia 8: 11-16.

Serrano I.L. 2010. Distribuiçāo e conservação de aves migratórias Neárticas da ordem Charadriiformes (famílias Charadriidae $e$ Scolopacidae) no Brasil. PhD. Thesis. Belém: Universidade Federal do Pará.

Skagen S.K. 2006. Migration stopovers and the conservation of Arctic-breeding Calidridine sandpipers. Auk 123: 313-323.

SNA (Sistema Nacional de Anilhamento de Aves Silvestres). 2019. Calidris pusilla. Banco de Dados CEMAVE/ICMBio. https://snaint.ibama.gov.br/ (Restrict access data).

Stotz D.F., Bierregaard R.O., Cohn-Haft M., Petermann P., Smith J., Whittaker A. \& Wilson S.V. 1992. The status of North American migrants in central Amazonian Brazil. Condor 94: 608-621.

Toledo L.F. 2019. FNJV - Fonoteca Neotropical Jacques Vielliard, v. 1.33. Universidade Estadual de Campinas - Instituto de Biologia. https://www.gbif.org (Access on 21 August 2019).

Tubelis D.P. \& Tomas W.M. 2003. Bird species of the Pantanal wetland, Brazil. Ararajuba 11: 5-37.

WikiAves. 2019. Calidris pusilla. A Enciclópedia de Aves do Brasil (Online). http://www.wikiaves.com (Access on 21 August 2019).

Associate Editor: Marcos P. Dantas. 